\title{
Fractional Brownian sheet and martingale difference random fields
}

\author{
Zhi Wang $^{1 *}$ and Jing Cui ${ }^{2}$
}

${ }^{\text {*Correspondence: }}$

wangzhi1006@hotmail.com

'School of Sciences, Ningbo

University of Technology,

201 Fenghua Rd., Ningbo, 315211, P.R. China

Full list of author information is

available at the end of the article

\begin{abstract}
In this paper, we prove a functional central limit theorem for the multidimensional parameter fractional Brownian sheet using martingale difference random fields. The proof is based on the invariance principle for the Brownian sheet due Poghosyan and Roelly (Stat. Probab. Lett. 38:235-245, 1998).
\end{abstract}

MSC: 60B10; 60G15

Keywords: martingale difference random fields; multidimensional parameter fractional Brownian sheet; weak convergence

\section{Introduction}

Self-similar stochastic processes with long range dependence (or long memory) are important aspect of stochastic models in various scientific areas, such as econometrics, network traffic analysis, hydrology, telecommunications, and so on. These are processes $X=\left\{X_{t}, t \geq 0\right\}$ whose dependence on the time parameter $t$ is self-similar, in the sense that there exists a (self-similarity) parameter $0<H<1$ such that for any constant $c \geq 0$, $\left\{X_{c t}, t \geq 0\right\}$ and $\left\{c^{H} X_{t}, t \geq 0\right\}$ have the same distribution. These processes are often endowed with other distinctive properties.

Fractional Brownian motion ( $\mathrm{fBm}$ ) is the usual candidate to model phenomena in which the self-similarity property can be observed from the empirical data. It is a suitable generalization of the standard Brownian motion $B$, which exhibits a long range dependence (when $H>1 / 2$ ), self-similarity, and Hölder's continuity, and which has stationary increments. Some surveys and comprehensive literature concerning $\mathrm{fBm}$ could be found in Biagini et al. [2], Gradinaru et al. [3], Hu [4], Mishura [5] and Nualart [6].

The so-called fBm of Hurst parameter $H$ is a continuous centered Gaussian process $B^{H}=\left\{B_{t}^{H}, t \geq 0\right\}$ with the covariance function

$$
R(t, s)=E\left[B_{t}^{H} B_{s}^{H}\right]=\frac{1}{2}\left[s^{2 H}+t^{2 H}-|t-s|^{2 H}\right] .
$$

Recall that $B^{H}$ has the following integral representation with respect to the standard Brownian motion $B$ (when $H>\frac{1}{2}$ ):

$$
B_{t}^{H}=\int_{0}^{t} K_{H}(t, s) d B_{s}, \quad t \geq 0,
$$

(0) 2016 Wang and Cui. This article is distributed under the terms of the Creative Commons Attribution 4.0 International License (http://creativecommons.org/licenses/by/4.0/), which permits unrestricted use, distribution, and reproduction in any medium, provided you give appropriate credit to the original author(s) and the source, provide a link to the Creative Commons license, and indicate if changes were made. 
where $K_{H}$ is the kernel defined by (see, e.g., Decreusefond and Üstünel [7])

$$
K_{H}(t, s)=\left(H-\frac{1}{2}\right) c_{H} s^{\frac{1}{2}-H} \int_{s}^{t} u^{H-\frac{1}{2}}(u-s)^{H-\frac{3}{2}} d u
$$

with $c_{H}>0$ the following normalizing constant:

$$
c_{H}=\sqrt{\frac{2 H \Gamma\left(\frac{3}{2}-H\right)}{\Gamma\left(H+\frac{1}{2}\right) \Gamma(2-2 H)}} .
$$

There are two possible multidimensional parameter extensions of the $\mathrm{fBm}$. The first one is the Lévy fractional Brownian random field (see Ciesielski and Kamont [8]), and the second one is the anisotropic fractional Brownian random field introduced by Kamont [9] as a centered Gaussian process $B^{\alpha}=\left\{B_{t}^{\alpha}, t \in \mathbb{R}_{+}^{d}\right\}$ with covariance function given by

$$
E\left[B_{t}^{\alpha} B_{s}^{\alpha}\right]=\prod_{k=1}^{d} \frac{1}{2}\left[s_{k}^{2 \alpha_{k}}+t_{k}^{2 \alpha_{k}}-\left|t_{k}-s_{k}\right|^{2 \alpha_{k}}\right],
$$

where $\alpha=\left(\alpha_{1}, \alpha_{2}, \ldots, \alpha_{d}\right) \in(0,1)^{d}$. We will call it a $d$-parameter fractional Brownian sheet. For $\alpha_{1}=\alpha_{2}=\cdots=\alpha_{d}=\frac{1}{2}$, it coincides with the standard $d$-parameter Brownian sheet $W=\left\{W_{t}, t \in \mathbb{R}_{+}^{d}\right\}$. This process is null on the axes and has a continuous version.

It is well known that a martingale difference random field is extremely useful because it imposes much milder restrictions on the memory of the sequence than under independence, yet most limit theorems that hold for an independent sequence will also hold for a martingale difference random field. Limit theorems for martingale differences were studied for example by Dai et al. [10], Nahapetian [11], Nieminen [12], Poghosyan [13], Shen and Yan [14], Shen et al. [15], Wang et al. [16] and so on. In this work, we will present a multidimensional parameter invariance principle for the fractional Brownian sheet, which is proved by a convergence criterion for random fields to multi-parameter Brownian sheet proved in Poghosyan and Roelly [1].

The rest of this paper is organized as follows. Section 2 contains some preliminaries on the multidimensional parameter stochastic processes and a precise statement of the main result of this paper. Finally, Section 3 is devoted to a proof of the main weak convergence theorem, Theorem 2.1.

\section{Preliminaries and main results}

We will use the definitions and notations introduced in the basic work of Bickel and Wichura [17]. Consider $[0,1]^{d}$ with the usual partial order. Let $(\Omega, \mathscr{F}, P)$ be a complete probability space and let $\left\{\mathscr{F}_{t} ; t \in[0,1]^{d}\right\}$ be a family of sub- $\sigma$-fields of $\mathscr{F}$ such that $\mathscr{F}_{s} \subseteq \mathscr{F}_{t}$ for any $s \leq t$. Given $s \leq t$, we denote by $\Delta_{s} X_{t}$ the increment of the process $X$ over the rectangle $(s, t]=\prod_{i=1}^{d}\left(s_{i}, t_{i}\right] \subset \mathbb{R}^{d}$.

Let $\Lambda$ be the group of all mappings $\lambda:[0,1]^{d} \rightarrow[0,1]^{d}$ of the form $\lambda(t)=\left(\lambda_{1}\left(t_{1}\right), \ldots\right.$, $\left.\lambda_{d}\left(t_{d}\right)\right)$, where each $\lambda_{i}:[0,1] \rightarrow[0,1]$ is continuous, is strictly increasing, and fixes zero and one. Denote by $D=D\left([0,1]^{d}\right)$ the Skorohod space of functions on $[0,1]^{d}$ which are continuous from above with limits from below and equip $D$, as usual, with the metric

$$
d(x, y):=\inf \{\min (\|x-y \lambda\|,\|\lambda\|): \lambda \in \Lambda\}
$$


where $\|x-y \lambda\|=\sup \left\{|x(t)-y(\lambda(t))|: t \in[0,1]^{d}\right\}$ and $\|\lambda\|=\sup \left\{|\lambda(t)-t|: t \in[0,1]^{d}\right\}$. Under this metric, $D$ is a separable and complete metric space. For more details we refer to Bickel and Wichura [17]. Let $X, X^{n}$ be processes in $D$, we say $X^{n}$ converges in law to $X$ if $E f\left(X^{n}\right) \rightarrow$ $E f(X)$ for all bounded and continuous functions $f: D \rightarrow \mathbb{R}$ as $n$ tends to infinity.

On the $d$-dimensional integer lattice $\mathbb{Z}^{d}$, let $I$ be the $\sigma$-algebra of invariant subsets of $\Omega$ :

$$
I=\left\{A \in \mathscr{F}: \tau_{u}(A)=A \text { for each } u \in \mathbb{Z}^{d}\right\}
$$

where $\left\{\tau_{u}, u \in \mathbb{Z}^{d}\right\}$ is the group of translations, acting on $\Omega$ by $\tau_{u}(X)=X(t-u), t \in \mathbb{Z}^{d}$.

Definition 2.1 A random field $\left\{\xi(t), t \in \mathbb{Z}^{d}\right\}$ is called translation invariant (homogeneous) if $P\left(\tau_{u}(A)\right)=P(A)$ for each $A \in \mathscr{F}$ and $u \in \mathbb{Z}^{d}$.

Definition 2.2 A translation invariant random field $\left\{\xi(t), t \in \mathbb{Z}^{d}\right\}$ is called ergodic if $P$ is trivial on the $\sigma$-algebra of invariant subsets, i.e. $P(A)=0$ or $P(A)=1$ for each $A \in I$.

$$
\begin{aligned}
& \text { For } u=\left(u_{1}, u_{2}, \ldots, u_{d}\right) \in \mathbb{Z}^{d} \text {, let } \\
& \qquad \mathbb{Z}_{-}^{d}(u)=\left\{t \in \mathbb{Z}^{d}: \exists j, 1 \leq j \leq d \text { such that } t_{j}<u_{j}\right\}
\end{aligned}
$$

and let $\mathbb{Z}_{+}^{d}(u)=\mathbb{Z}^{d} \backslash \mathbb{Z}_{-}^{d}(u)$. For a random field $\left\{\xi(t), t \in \mathbb{Z}^{d}\right\}$, set $\mathcal{P}(u)=\sigma\left\{\xi(t), t \in \mathbb{Z}_{-}^{d}(u)\right\}$.

Definition 2.3 A random field $\left\{\xi(t), t \in \mathbb{Z}^{d}\right\}$ is called a martingale difference if, for each $t \in \mathbb{Z}^{d}$

$$
E(\xi(t) \mid \mathcal{P}(t-1))=0 \quad \text { a.s., }
$$

where $t-1=\left(t_{1}-1, t_{2}-1, \ldots, t_{d}-1\right)$.

Set

$$
K_{H}^{n}(t, s):=n \int_{s-\frac{1}{n}}^{s} K_{H}\left(\frac{\lfloor n t\rfloor}{n}, u\right) d u, \quad n=1,2, \ldots
$$

where $\lfloor x\rfloor$ denotes the greatest integer not exceeding $x$. It is an approximation of $K_{H}(t, s)$.

Let $\alpha_{k}>\frac{1}{2}, k=1,2, \ldots, d$. Taking into account the integral representation (1.1) for the $\mathrm{fBm}$, the $d$-parameter fractional Brownian sheet $B^{\alpha}$ has also the following integral representation:

$$
B_{t}^{\alpha}=\int_{0}^{t_{d}} \cdots \int_{0}^{t_{1}} K_{\alpha_{1}}\left(t_{1}, u_{1}\right) K_{\alpha_{2}}\left(t_{2}, u_{2}\right) \cdots K_{\alpha_{d}}\left(t_{d}, u_{d}\right) d W_{u}
$$

where $\left\{K_{\alpha_{k}}, k=1, \ldots, d\right\}$ is given by (1.2).

The following theorem is the main result of the present paper, it is a multidimensional extension of Nieminen [12].

Theorem 2.1 Let $\alpha_{k}>\frac{1}{2}, k=1,2, \ldots, d .\left\{\xi_{i_{1}, i_{2}, \ldots, i_{d}}^{n}, i_{k}=1,2, \ldots\right\}$ is a translation invariant, ergodic, martingale difference random field with finite second moment $E\left(\xi_{i_{1}, i_{2}, \ldots, i_{d}}\right)^{2}<+\infty$ 
such that

$$
\lim _{n \rightarrow \infty} \xi_{i_{1}, i_{2}, \ldots, i_{d}}^{n}=1 \quad \text { a.s. }
$$

for all $1 \leq i_{k} \leq n$ and

$$
\max _{1 \leq i_{k} \leq n}\left|\xi_{i_{1}, i_{2}, \ldots, i_{d}}^{n}\right| \leq C \quad \text { a.s. }
$$

for some $C \geq 1$. Define, for all $n \geq 1, t=\left(t_{1}, t_{2}, \ldots, t_{d}\right) \in[0,1]^{d}$,

$$
B_{t}^{n}:=\frac{1}{n^{\frac{d}{2}}} \sum_{k=1}^{d} \sum_{i_{k}=1}^{\left\lfloor n t_{k}\right\rfloor} \xi_{i_{1}, i_{2}, \ldots, i_{d}}^{n}
$$

and

$$
\begin{aligned}
Z_{t}^{n}:= & \int_{0}^{t_{d}} \cdots \int_{0}^{t_{1}} K_{\alpha_{1}}^{n}\left(t_{1}, u_{1}\right) \cdots K_{\alpha_{d}}^{n}\left(t_{d}, u_{d}\right) d B_{u}^{n} \\
= & n^{\frac{d}{2}} \sum_{k=1}^{d} \sum_{i_{k}=1}^{\left\lfloor n t_{k}\right\rfloor} \xi_{i_{1}, i_{2}, \ldots, i_{d}}^{n} \\
& \times \int_{\frac{i_{d}-1}{n}}^{\frac{i_{d}}{n}} \cdots \int_{\frac{i_{1}-1}{n}}^{\frac{i_{1}}{n}} K_{\alpha_{1}}\left(\frac{\left\lfloor n t_{1}\right\rfloor}{n}, u_{1}\right) \cdots K_{\alpha_{d}}\left(\frac{\left\lfloor n t_{d}\right\rfloor}{n}, u_{d}\right) d u_{1} \cdots d u_{d},
\end{aligned}
$$

where the kernel $K_{\alpha_{k}}$ is given by (1.2) and the sequence $\left\{K_{\alpha_{k}}^{n}, n=1,2, \ldots\right\}$ defined by (2.1) is an approximation of $K_{\alpha_{k}}$.

Then, $\left\{Z^{n}\right\}$ converges weakly in the Skorohod space $D\left([0,1]^{d}\right)$ to the d-parameter fractional Brownian sheet $B^{\alpha}$.

In the rest of this paper, most of the estimates contain unspecified constants. An unspecified positive and finite constant will be denoted by $C$, which may not be the same in each occurrence. Sometimes we shall emphasize the dependence of these constants upon parameters.

\section{Proof of Theorem 2.1}

In this section, we will prove Theorem 2.1. We verify weak convergence via the convergence of finite dimensional distributions and tightness. We first check the tightness. Since the $Z^{n}$ are null on the axes, using the criterion established in Bickel and Wichura [17], it suffices to prove the following lemma.

Lemma 3.1 Let $\left\{Z_{t}^{n}\right\}$ be the family of processes defined by (2.6). Then for any $s, t \in[0,1]^{d}$ with $s<t$ and any even number $m \geq 2$, there exists a constant $C_{m}$ such that

$$
\sup _{n} E\left(\Delta_{s} Z_{t}^{n}\right)^{m} \leq C_{m} \prod_{k=1}^{d}\left(t_{k}-s_{k}\right)^{m \alpha_{k}}
$$


Proof Notice that

$$
\begin{aligned}
\Delta_{s} Z_{t}^{n}= & \int_{s_{d}}^{t_{d}} \cdots \int_{s_{1}}^{t_{1}}\left(K_{\alpha_{1}}^{n}\left(t_{1}, u_{1}\right)-K_{\alpha_{1}}^{n}\left(s_{1}, u_{1}\right)\right) \times \cdots \times\left(K_{\alpha_{d}}^{n}\left(t_{d}, u_{d}\right)-K_{\alpha_{1}}^{n}\left(s_{d}, u_{d}\right)\right) d B_{u}^{n} \\
= & n^{\frac{d}{2}} \sum_{k=1}^{d} \sum_{i_{k}=1}^{\left\lfloor n t_{k}\right\rfloor} \xi_{i_{1}, i_{2}, \ldots, i_{d}}^{n} \int_{\frac{i_{d}-1}{n}}^{\frac{i_{d}}{n}} \cdots \int_{\frac{i_{1}-1}{n}}^{\frac{i_{1}}{n}}\left(K_{\alpha_{1}}\left(\frac{\left\lfloor n t_{1}\right\rfloor}{n}, u_{1}\right)-K_{\alpha_{1}}\left(\frac{\left\lfloor n s_{1}\right\rfloor}{n}, u_{1}\right)\right) \\
& \times \cdots \times\left(K_{\alpha_{d}}\left(\frac{\left\lfloor n t_{d}\right\rfloor}{n}, u_{d}\right)-K_{\alpha_{d}}\left(\frac{\left\lfloor n s_{d}\right\rfloor}{n}, u_{d}\right)\right) d u_{1} \cdots d u_{d} \\
= & n^{\frac{d}{2}} \sum_{k=1}^{d} \sum_{i_{k}=1}^{\left\lfloor n t_{k}\right\rfloor} \xi_{i_{1}, i_{2}, \ldots, i_{d}}^{n} \int_{\frac{i_{1}-1}{n}}^{\frac{i_{1}}{n}}\left(K_{\alpha_{1}}\left(\frac{\left\lfloor n t_{1}\right\rfloor}{n}, u_{1}\right)-K_{\alpha_{1}}\left(\frac{\left\lfloor n s_{1}\right\rfloor}{n}, u_{1}\right)\right) d u_{1} \\
& \times \cdots \times \int_{\frac{i_{d}-1}{n}}^{\frac{i_{d}}{n}}\left(K_{\alpha_{d}}\left(\frac{\left\lfloor n t_{d}\right\rfloor}{n}, u_{d}\right)-K_{\alpha_{d}}\left(\frac{\left\lfloor n s_{d}\right\rfloor}{n}, u_{d}\right)\right) d u_{d} .
\end{aligned}
$$

Thus,

$$
\begin{aligned}
E\left(\Delta_{s} Z_{t}^{n}\right)^{m}= & n^{\frac{d m}{2}} E\left[\sum_{k=1}^{d} \sum_{i_{k}=1}^{\left\lfloor n t_{k}\right\rfloor} \xi_{i_{1}, i_{2}, \ldots, i_{d}}^{n} \int_{\frac{i_{1}-1}{n}}^{\frac{i_{1}}{n}}\left(K_{\alpha_{1}}\left(\frac{\left\lfloor n t_{1}\right\rfloor}{n}, u_{1}\right)-K_{\alpha_{1}}\left(\frac{\left\lfloor n s_{1}\right\rfloor}{n}, u_{1}\right)\right) d u_{1}\right. \\
& \left.\times \cdots \times \int_{\frac{i_{d}-1}{n}}^{\frac{i_{d}}{n}}\left(K_{\alpha_{d}}\left(\frac{\left\lfloor n t_{d}\right\rfloor}{n}, u_{d}\right)-K_{\alpha_{d}}\left(\frac{\left\lfloor n s_{d}\right\rfloor}{n}, u_{d}\right)\right) d u_{d}\right]^{m} \\
\leq & n^{\frac{d m}{2}} C_{m}\left[\sum_{k=1}^{d} \sum_{i_{k}=1}^{\left\lfloor n t_{k}\right\rfloor} \int_{\frac{i_{1}-1}{n}}^{\frac{i_{1}}{n}}\left(K_{\alpha_{1}}\left(\frac{\left\lfloor n t_{1}\right\rfloor}{n}, u_{1}\right)-K_{\alpha_{1}}\left(\frac{\left\lfloor n s_{1}\right\rfloor}{n}, u_{1}\right)\right) d u_{1}\right. \\
& \left.\times \cdots \times \int_{\frac{i_{d}-1}{n}}^{\frac{i_{d}}{n}} \ldots\left(K_{\alpha_{d}}\left(\frac{\left\lfloor n t_{d}\right\rfloor}{n}, u_{d}\right)-K_{\alpha_{d}}\left(\frac{\left\lfloor n s_{d}\right\rfloor}{n}, u_{d}\right)\right) d u_{d}\right]^{m} \\
= & C_{m}\left[\prod_{k=1}^{d}\left(\sqrt{n} \sum_{i_{k}=1}^{\left\lfloor n t_{k}\right\rfloor} \int_{\frac{i_{k-1}}{n}}^{\frac{i_{k}}{n}}\left(K_{\alpha_{k}}\left(\frac{\left\lfloor n t_{k}\right\rfloor}{n}, u_{k}\right)-K_{\alpha_{k}}\left(\frac{\left\lfloor n s_{k}\right\rfloor}{n}, u_{k}\right)\right) d u_{k}\right)^{2}\right]^{\frac{m}{2}} .
\end{aligned}
$$

By the Cauchy-Schwarz inequality, the last expression can be bounded by

$$
\begin{aligned}
& C_{m} \prod_{k=1}^{d}\left[\sum_{i_{k}=1}^{\left\lfloor n t_{k}\right\rfloor} \int_{\frac{i_{k-1}}{n}}^{\frac{i_{k}}{n}}\left(K_{\alpha_{k}}\left(\frac{\left\lfloor n t_{k}\right\rfloor}{n}, u_{k}\right)-K_{\alpha_{k}}\left(\frac{\left\lfloor n s_{k}\right\rfloor}{n}, u_{k}\right)\right)^{2} d u_{k}\right]^{\frac{m}{2}} \\
& \leq C_{m} \prod_{k=1}^{d}\left[\int_{0}^{t_{k}}\left(K_{\alpha_{k}}\left(\frac{\left\lfloor n t_{k}\right\rfloor}{n}, u_{k}\right)-K_{\alpha_{k}}\left(\frac{\left\lfloor n s_{k}\right\rfloor}{n}, u_{k}\right)\right)^{2} d u_{k}\right]^{\frac{m}{2}} \\
& \quad=C_{m} \prod_{k=1}^{d}\left\lfloor\left.\frac{\left\lfloor n t_{k}\right\rfloor-\left\lfloor n s_{k}\right\rfloor}{n}\right|^{m \alpha_{k}} .\right.
\end{aligned}
$$

Let us have now arbitrary $0<s_{k}<t_{k}$ and $\frac{1}{2}<\alpha_{k}<1$. If $n t_{k}-n s_{k} \geq 1$, then we have $\left|\frac{\left\lfloor n t_{k}\right\rfloor-\left\lfloor n s_{k}\right\rfloor}{n}\right|^{2 \alpha_{k}} \leq\left|2\left(t_{k}-s_{k}\right)\right|^{2 \alpha_{k}}$. On the other hand, if $n t_{k}-n s_{k}<1$ then either $t_{k}$ and $s_{k}$ belong to a same subinterval $\left[\frac{m}{n}, \frac{m+1}{n}\right)$ for some integer $m$, which implies $\left|\frac{\left\lfloor n t_{k}\right\rfloor-\left\lfloor n s_{k}\right\rfloor}{n}\right|^{2 \alpha_{k}}=0$. 
Therefore, we get

$$
\left|\frac{\left\lfloor n t_{k}\right\rfloor-\left\lfloor n s_{k}\right\rfloor}{n}\right|^{2 \alpha_{k}} \leq\left|2\left(t_{k}-s_{k}\right)\right|^{2 \alpha_{k}}
$$

for all $n \geq 1$. This completes the proof of this lemma.

We now proceed with the identification of the limit law by proving the convergence of the finite-dimensional distributions of the processes $\left\{Z_{t}^{n}\right\}$ to those of $B^{\alpha}$.

Theorem 3.1 The family of processes $\left\{Z_{t}^{n}\right\}$ defined by (2.6) converges, in the sense of a finite-dimensional distribution, to the d-parameter fractional Brownian sheet $B^{\alpha}$.

Proof For all $N \in \mathbb{N}$, consider $a_{1}, \ldots, a_{N} \in \mathbb{R}$ and $t^{1}, \ldots, t^{N} \in[0,1]^{d}$. It suffices to prove that the linear combination

$$
Y^{n}:=\sum_{j=1}^{N} a_{j} Z_{t^{j}}^{n}
$$

converges in distribution, as $n$ tends to infinity, to a normally distributed random variable with zero mean and variance

$$
E\left(\sum_{j=1}^{N} a_{j} B_{t^{j}}^{\alpha}\right)^{2}
$$

Fact is that the zero mean is trivial. Next, we observe that

$$
\begin{aligned}
\left(\sigma^{n}\right)^{2}:= & E\left(Y^{n}\right)^{2}=\sum_{j, l=1}^{N} a_{j} a_{l} E Z_{t j}^{n} Z_{t^{l}}^{n} \\
= & \sum_{j, l=1}^{N} a_{j} a_{l} n^{d} \sum_{k=1}^{d} \sum_{i_{k}=1}^{n} \int_{\frac{i_{d}-1}{n}}^{\frac{i_{d}}{n}} \cdots \int_{\frac{i_{1}-1}{n}}^{\frac{i_{1}}{n}} K_{\alpha_{1}}\left(\frac{\left\lfloor n t_{1}^{j}\right\rfloor}{n}, u_{1}\right) \\
& \times \cdots \times K_{\alpha_{d}}\left(\frac{\left\lfloor n t_{d}^{j}\right\rfloor}{n}, u_{d}\right) d u_{1} \cdots d u_{d} \\
& \times \int_{\frac{i_{d}-1}{n}}^{\frac{i_{d}}{n}} \cdots \int_{\frac{i_{1}-1}{n}}^{\frac{i_{1}}{n}} K_{\alpha_{1}}\left(\frac{\left\lfloor n t_{1}^{l}\right\rfloor}{n}, u_{1}\right) \cdots K_{\alpha_{d}}\left(\frac{\left\lfloor n t_{d}^{l}\right\rfloor}{n}, u_{d}\right) d u_{1} \cdots d u_{d}\left(\xi_{i_{1}, i_{2}, \ldots, i_{d}}^{n}\right)^{2} \\
= & \sum_{j, l=1}^{N} a_{j} a_{l} n^{d} \prod_{k=1}^{d} \sum_{i_{k}=1}^{n} \int_{\frac{i_{k}-1}{n}}^{\frac{i_{k}}{n}} K_{\alpha_{k}}\left(\frac{\left\lfloor n t_{k}^{j}\right\rfloor}{n}, u\right) d u \int_{\frac{i_{k}-1}{n}}^{\frac{i_{k}}{n}} K_{\alpha_{k}}\left(\frac{\left\lfloor n t_{k}^{l}\right\rfloor}{n}, u\right) d u\left(\xi_{i_{1}, i_{2}, \ldots, i_{d}}^{n}\right)^{2} .
\end{aligned}
$$

Consider now the inner sum. By the mean value theorem, we have

$$
\begin{gathered}
n \sum_{i_{k}=1}^{n} \int_{\frac{i_{k}-1}{n}}^{\frac{i_{k}}{n}} K_{\alpha_{k}}\left(\frac{\left\lfloor n t_{k}^{j}\right\rfloor}{n}, u\right) d u \int_{\frac{i_{k}-1}{n}}^{\frac{i_{k}}{n}} K_{\alpha_{k}}\left(\frac{\left\lfloor n t_{k}^{l}\right\rfloor}{n}, u\right) d u \\
=\frac{1}{n} \sum_{i_{k}=1}^{n} K_{\alpha_{k}}\left(\frac{\left\lfloor n t_{k}^{j}\right\rfloor}{n}, u_{i, k}^{n}\right) K_{\alpha_{k}}\left(\frac{\left\lfloor n t_{k}^{l}\right\rfloor}{n}, u_{i, l}^{n}\right)
\end{gathered}
$$


for some $u_{i, k}^{n}, u_{i, l}^{n} \in\left(\frac{i_{k}-1}{n}, \frac{i_{k}}{n}\right]$. Since the kernel $K_{\alpha_{k}}(t, \cdot)$ is continuous and decreasing we see that (3.1) is equal to

$$
\frac{1}{n} \sum_{i_{k}=1}^{n} K_{\alpha_{k}}\left(\frac{\left\lfloor n t_{k}^{j}\right\rfloor}{n}, u_{i}^{n}\right) K_{\alpha_{k}}\left(\frac{\left\lfloor n t_{k}^{l}\right\rfloor}{n}, u_{i}^{n}\right)
$$

for some

$$
u_{i}^{n} \in\left[\min \left(u_{i, k}^{n}, u_{i, l}^{n}\right), \max \left(u_{i, k}^{n}, u_{i, l}^{n}\right)\right] \subseteq\left(\frac{i_{k}-1}{n}, \frac{i_{k}}{n}\right] .
$$

On the other hand, we observe that the kernel $K_{H}$ with $\frac{1}{2}<H<1$ is continuous with respect to both arguments and the maps $t \mapsto \frac{\lfloor n t\rfloor}{n}$ converge uniformly to the identity map in $[0, T]$. So (3.2) is a Riemann type sum. Thus, combining with (2.3), we see that (3.1) converges to

$$
\int_{0}^{1} K_{\alpha_{k}}\left(t_{k}^{j}, u\right) K_{\alpha_{k}}\left(t_{k}^{l}, u\right) d u
$$

As a consequence, we see that $\left(\sigma^{n}\right)^{2}$ converges to

$$
\sum_{j, l=1}^{N} a_{j} a_{l} \prod_{k=1}^{d} \int_{0}^{1} K_{\alpha_{k}}\left(t_{k}^{j}, u\right) K_{\alpha_{k}}\left(t_{k}^{l}, u\right) d u=E\left(\sum_{j=1}^{N} a_{j} B_{t^{j}}^{\alpha}\right)^{2} .
$$

Let us now write $Y^{n}$ as

$$
\begin{aligned}
Y^{n}= & \sum_{j=1}^{N} a_{j} Z_{t^{j}}^{n} \\
= & \sum_{k=1}^{d} \sum_{i_{k}=1}^{n} n^{\frac{d}{2}} \xi_{i_{1}, i_{2}, \ldots, i_{d}} \sum_{j=1}^{N} a_{j} \int_{\frac{i_{d}-1}{n}}^{\frac{i_{d}}{n}} \cdots \int_{\frac{i_{1}-1}{n}}^{\frac{i_{1}}{n}} K_{\alpha_{1}}\left(\frac{\left\lfloor n t_{1}^{j}\right\rfloor}{n}, u_{1}\right) \\
& \times \cdots \times K_{\alpha_{d}}\left(\frac{\left\lfloor n t_{d}^{j}\right\rfloor}{n}, u_{d}\right) d u_{1} \cdots d u_{d} \\
:= & \sum_{k=1}^{d} \sum_{i_{k}=1}^{n} Y_{i_{1}, i_{2}, \ldots, i_{d} .}^{n}
\end{aligned}
$$

Then, it remains to prove that the following Lindeberg condition is satisfied:

$$
\lim _{n \rightarrow \infty} \sum_{k=1}^{d} \sum_{i_{k}=1}^{n} E\left[\left(Y_{i_{1}, i_{2}, \ldots, i_{d}}^{n}\right)^{2} 1_{\left\{\left|Y_{i_{1}, i_{2}, \ldots, i_{d}}^{n}\right|>\varepsilon\right\}} \mid \mathcal{P}\left(i_{1}-1, i_{2}-1, \ldots, i_{d}-1\right)\right]=0
$$

for all $\varepsilon>0$. By the Cauchy-Schwarz inequality and the fact that the kernel $K_{H}(t, s)$ with $\frac{1}{2}<H<1$ is increasing in $t$ and decreasing in $s$, we have

$$
\begin{aligned}
\left(Y_{i_{1}, i_{2}, \ldots, i_{d}}^{n}\right)^{2}= & n^{d}\left(\xi_{i_{1}, i_{2}, \ldots, i_{d}}^{n}\right)^{2} \\
& \times\left(\sum_{j=1}^{N} a_{j} \int_{\frac{i_{d}-1}{n}}^{\frac{i_{d}}{n}} \cdots \int_{\frac{i_{1}-1}{n}}^{\frac{i_{1}}{n}} K_{\alpha_{1}}\left(\frac{\left\lfloor n t_{1}^{j}\right\rfloor}{n}, u_{1}\right) \cdots K_{\alpha_{d}}\left(\frac{\left\lfloor n t_{d}^{j}\right\rfloor}{n}, u_{d}\right) d u_{1} \cdots d u_{d}\right)^{2}
\end{aligned}
$$




$$
\begin{aligned}
& \leq n^{d}\left(\xi_{i_{1}, i_{2}, \ldots, i_{d}}^{n}\right)^{2} A\left(\int_{\frac{i_{1}-1}{n}}^{\frac{i_{1}}{n}} K_{\alpha_{1}}\left(1, u_{1}\right) d u_{1} \times \cdots \times \int_{\frac{i_{d}-1}{n}}^{\frac{i_{d}}{n}} K_{\alpha_{d}}\left(1, u_{d}\right) d u_{d}\right)^{2} \\
& \leq\left(\xi_{i_{1}, i_{2}, \ldots, i_{d}}^{n}\right)^{2} A \int_{\frac{i_{1}-1}{n}}^{\frac{i_{1}}{n}} K_{\alpha_{1}}^{2}\left(1, u_{1}\right) d u_{1} \times \cdots \times \int_{\frac{i_{d}-1}{n}}^{\frac{i_{d}}{n}} K_{\alpha_{d}}^{2}\left(1, u_{d}\right) d u_{d} \\
& \leq\left(\xi_{i_{1}, i_{2}, \ldots, i_{d}}^{n}\right)^{2} A \prod_{k=1}^{d} \int_{0}^{\frac{1}{n}} K_{\alpha_{k}}^{2}(1, u) d u \\
& =\left(\xi_{i_{1}, i_{2}, \ldots, i_{d}}^{n}\right)^{2} A \delta^{n},
\end{aligned}
$$

where $A:=\left(\sum_{j=1}^{N} a_{j}\right)^{2}$ and $\delta^{n}:=\prod_{k=1}^{d} \int_{0}^{\frac{1}{n}} K_{\alpha_{k}}^{2}(1, u) d u$. So we get

$$
\left\{\left|Y_{i_{1}, i_{2}, \ldots, i_{d}}^{n}\right|>\varepsilon\right\}=\left\{\left(Y_{i_{1}, i_{2}, \ldots, i_{d}}^{n}\right)^{2}>\varepsilon^{2}\right\} \subseteq\left\{\left(\xi_{i_{1}, i_{2}, \ldots, i_{d}}^{n}\right)^{2} A \delta^{n}>\varepsilon^{2}\right\} .
$$

Consequently, we obtain

$$
\begin{aligned}
& E\left[\left(Y_{i_{1}, i_{2}, \ldots, i_{d}}^{n}\right)^{2} 1_{\left\{\left|Y_{i_{1}, i_{2}, \ldots, i_{d}}^{n}\right|>\varepsilon\right\}} \mid \mathcal{P}\left(i_{1}-1, i_{2}-1, \ldots, i_{d}-1\right)\right] \\
& \quad \leq E\left[\left(\xi_{i_{1}, i_{2}, \ldots, i_{d}}^{n}\right)^{2} A \delta^{n} 1_{\left\{\left(\xi_{i_{1}, i_{2}, \ldots, i_{d}}^{n}\right)^{2} A \delta^{n}>\varepsilon^{2}\right\}} \mid \mathcal{P}\left(i_{1}-1, i_{2}-1, \ldots, i_{d}-1\right)\right] \\
& \quad \leq C A \delta^{n} E\left[1_{\left\{\left(\xi_{i_{1}, i_{2}, \ldots, i_{d}}^{n}\right)^{2} A \delta^{n}>\varepsilon^{2}\right\}} \mid \mathcal{P}\left(i_{1}-1, i_{2}-1, \ldots, i_{d}-1\right)\right]
\end{aligned}
$$

for all $i_{k}=1,2, \ldots, n, k=1,2, \ldots, d$, and that

$$
\begin{aligned}
& \sum_{k=1}^{d} \sum_{i_{k}=1}^{n} E\left[\left(Y_{i_{1}, i_{2}, \ldots, i_{d}}^{n}\right)^{2} 1_{\left\{\left|Y_{i_{1}, i_{2}, \ldots, i_{d}}^{n}\right|>\varepsilon\right\}} \mid \mathcal{P}\left(i_{1}-1, i_{2}-1, \ldots, i_{d}-1\right)\right] \\
& \leq \sum_{k=1}^{d} \sum_{i_{k}=1}^{n} C A \delta^{n} E\left[1_{\left\{\left(\xi_{i_{1}, i_{2}, \ldots, i_{d}}^{n}\right)^{2} A \delta^{n}>\varepsilon^{2}\right\}} \mid \mathcal{P}\left(i_{1}-1, i_{2}-1, \ldots, i_{d}-1\right)\right] \\
& \leq C A \delta^{n} \sum_{k=1}^{d} \sum_{i_{k}=1}^{n} E\left[1_{\left\{C A \delta^{n}>\varepsilon^{2}\right\}}\right] \rightarrow 0 \quad(n \rightarrow \infty)
\end{aligned}
$$

because $\delta^{n} \rightarrow 0$, implies $1_{\left\{C A \delta^{n}>\varepsilon^{2}\right\}} \rightarrow 0$.

Thus, the Lindeberg condition (3.3) holds and the proof of Theorem 2.1 is now complete.

\section{Competing interests}

The authors declare that they have no competing interests.

\section{Authors' contributions}

All authors read and approved the final manuscript.

\section{Author details}

'School of Sciences, Ningbo University of Technology, 201 Fenghua Rd., Ningbo, 315211, P.R. China. ${ }^{2}$ Department of Statistics, Anhui Normal University, East Beijing Rd., Wuhu, 241000, P.R. China.

\section{Acknowledgements}

This work was supported by National Natural Science Foundation-Mathematical Tianyuan Foundation of China (11526117), National Natural Science Foundation of China (11401010, 11401313, 11401093, 61503205), Zhejiang Provincial Natural Science Foundation (LQ16A010006) and the Natural Science Foundation of Ningbo Municipality (2015A610158).

Received: 27 March 2016 Accepted: 11 August 2016 Published online: 22 August 2016 
References

1. Poghosyan, S, Roelly, S: Invariance principle for martingale-difference random fields. Stat. Probab. Lett. 38, 235-245 (1998)

2. Biagini, F, Hu, Y, Øksendal, B, Zhang, T: Stochastic Calculus for fBm and Applications. Probability and Its Applications. Springer, Berlin (2008)

3. Gradinaru, M, Nourdin, I, Russo, F, Vallois, P: $m$-Order integrals and generalized Itô's formula; the case of a fBm with any Hurst index. Ann. Inst. Henri Poincaré Probab. Stat. 41, 781-806 (2005)

4. $\mathrm{Hu}, \mathrm{Y}$ : Integral transformations and anticipative calculus for fractional Brownian motions. Mem. Am. Math. Soc. 175, 825 (2005)

5. Mishura, YS: Stochastic Calculus for Fractional Brownian Motion and Related Processes. Lect. Notes in Math., vol. 1929 (2008)

6. Nualart, D: Malliavin Calculus and Related Topics, 2nd edn. Springer, New York (2006)

7. Decreusefond, L, Üstünel, AS: Stochastic analysis of the fractional Brownian motion. Potential Anal. 10, 177-214 (1999)

8. Ciesielski, Z, Kamont, A: Lévy's fractional Brownian random field and function spaces. Acta Sci. Math. 60, 99-118 (1995)

9. Kamont, A: On the fractional anisotropic Wiener field. Probab. Math. Stat. 16, 85-98 (1996)

10. Dai, H, Hu, T, Lee, J: Operator fractional Brownian motion and martingale difference. Abstr. Appl. Anal. 2014, Article ID 791537 (2014)

11. Nahapetian, B: Billingsley-lbragimov theorem for martingale-difference random fields and its applications to some models of statistical physics. C. R. Acad. Sci. Paris, Ser. I. 320, 1539-1544 (1995)

12. Nieminen, A: Fractional Brownian motion and martingale-differences. Stat. Probab. Lett. 70, 1-10 (2004)

13. Poghosyan, S: Functional central limit theorem for martingale-difference random field. Izv. Nat. Akad. Nauk Armenii Math. 30, 137-154 (1995)

14. Shen, G, Yan, L: An approximation of subfractional Brownian motion. Commun. Stat., Theory Methods 43, 1873-1886 (2014)

15. Shen, G, Yin, X, Yan, L: Approximation of the Rosenblatt sheet. Mediterr. J. Math. (2016) doi:10.1007/s00009-015-0576-5

16. Wang, Z, Yan, L, Yu, X: Weak convergence to the fractional Brownian sheet using martingale differences. Stat. Probab. Lett. 92, 72-78 (2014)

17. Bickel, PJ, Wichura, MJ: Convergence criteria for multiparameter stochastic processes and some applications. Ann. Math. Stat. 42, 1656-1670 (1971)

\section{Submit your manuscript to a SpringerOpen ${ }^{\ominus}$ journal and benefit from:}

- Convenient online submission

- Rigorous peer review

- Immediate publication on acceptance

- Open access: articles freely available online

- High visibility within the field

- Retaining the copyright to your article 